\title{
A new geomagnetic index reveals the link between geomagnetic activity and human health
}

\author{
Hiroshi Morimoto
}

\begin{abstract}
The relationship between geomagnetic storms and human health has been debated for decades. The idea that the geomagnetic fluctuations may cause human ill health has implications for a wide range of illness from cardiovascular disease to mental illness. However, this has not been established in the literature. This work, using a new measure of geomagnetic activity, studies the associations between geomagnetic activity and human health. We classified magnetic storms into two categories, storms of sudden commencement and storms of gradual commencement, and counted their frequencies to get new indices called FSSC and FSGC respectively. We explored the correlation of these new indices with 16 leading causes of death in Japan from 1952 to 2004. We also compared our results with known indices of solar activity viz sunspot numbers and K-index. Significant positive correlations were between FSSC and Hypertensive diseases, Cerebrovascular diseases, Accidents, Transport accidents Malignant neoplasms, Diabetes mellitus, Heart diseases, Pneumonia, Chronic bronchitis and emphysema, Renal failure, and negative correlations between FSSC and Hypertensive diseases, Cerebrovascular diseases, Asthma, Gastric ulcer and duodenal ulcer, Senility, Accidents, Transport accidents. No correlations were seen between disease and sunspot numbers. Our new index contributed to revealing the link between the geomagnetic activity and human health more precisely than established indices.
\end{abstract}

Index Terms - geomagnetic storm, geomagnetic fluctuation, mortality, heart disease, transport accidents.

\section{INTRODUCTION}

The relationship between human health and the activity of the Sun has been discussed for many decades. The effect of geomagnetic fluctuation on human illness ranges from cardiovascular disease to mental illness. Research undertaken to assess the relationship between fluctuations in solar activity and human health (also known as heliobiology) has been reported by Palmer et al (2006) [1]. An increase in female psychotic depression has been shown following geomagnetic storms [2].

However, a significant correlation has not been established between geomagnetic storm levels and patients numbers ([2]), while the effects of weather on human health have been clearly studied [3].

Conversely, contradictory reports exist on both a decrease and an increase in the number of patients after geomagnetic storms $[4,5]$.
For instance, an increase in the incidence of sudden cardiac deaths has been observed after geomagnetic storms [4]; so has a decrease in the incidence of sudden cardiac deaths [5]. The Earth's magnetic field provides a protective shield against cosmic rays and prevents the atmosphere from being eroded by solar wind. Therefore, an increase in sudden cardiac deaths may be as a result of cosmic rays. Sunspot numbers and $\mathrm{K}$-index are known measures of assessing the sun's activity and the earth's magnetic field respectively. In order to establish the link between geomagnetic activity and human health, it is important to find a clear measure of fluctuations in geomagnetic activity from a point of view of human health.

This work studies the associations between geomagnetic activity and human health. A geomagnetic storm (commonly referred to as a solar storm) is a temporary disturbance in the Earth's magnetosphere caused by a solar wind shock wave and/or a cloud of magnetic field that interacts with the Earth's magnetic field. The disturbance in the interplanetary medium that drives the storm may result from a solar coronal mass ejection (CME) or a high speed stream (co-rotating interaction region or CIR) of the solar wind originating from a region of weak magnetic field on the Sun's surface. CME driven storms are more common during the maximum of the solar cycle, while CIR driven storms are more common during the minimum of the solar cycle. This work focuses on these differences in magnetic storms with a view to deduce new indices.

A geomagnetic storm is defined by extreme changes in geomagnetic disturbances, such as the K-index [6]. The geomagnetic $\mathrm{K}$-index is a measure of the disturbance level of the Earth's magnetic field. It is derived from the maximum fluctuations of horizontal components observed on a magnetometer during a three-hour interval.

A geomagnetic storm has three phases: the initial, main and recovery phases. The initial phase is characterized by a sudden increase in geomagnetic index, and is referred to as a storm of sudden commencement (ssc). Geomagnetic storms without an initial phase are called storms of gradual commencement $(\mathrm{sgc})$. The events of ssc and sgc have been recorded at Kakioka Magnetic Observatory in Japan. From the frequencies of these events of ssc and sgc observed at Kakioka, we formed new indices FSSC (Frequencies of Storm of Sudden Commencement) and FSGC (Frequencies of Storms of Gradual Commencement).

Using these indices, we explored the relationship between these indices and 16 leading causes of death in Japan between 1952 and 2004 (including heart disease and transport accidents) to identify links between the geomagnetic activity and human health that have not been illustrated by sunspot numbers and $\mathrm{K}$-index. 


\section{METHODS}

\section{A. Mortality data}

We obtained data on death rate from the Ministry of Health, Labor and Welfare in Japan that provided data on leading causes of death from 1899 to 2004.

The death rate was defined as the number of deaths per 100,000 persons in Japan. The original yearly data were available data from 1899 to 2004. Data from 1944 through 1946 were not available due to the second world war. Therefore only data from 1952 to 2004 were used in the interests of reliability.

The data on 16 leading causes of death were extracted from the Statistic Bureau, Ministry of Internal Affairs and Communications. These were comprised of data on : Tuberculosis, Malignant neoplasms, Diabetes mellitus, Hypertensive diseases, Heart diseases (Excluding hypertensive heart diseases), Cerebrovascular diseases, Pneumonia, Chronic bronchitis and emphysema, Asthma, Gastric ulcer and duodenal ulcer, Diseases of liver, Renal failure, Senility, Accidents, Transport accidents, and Suicide.

\section{B. $K$-indices}

To obtain data on the K-index, we relied on data supplied by Kakioka magnetic observatory that is maintained by the Japan Meteorological Agency. The geomagnetic observatory is

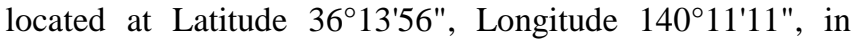
Ibaraki prefecture. The $\mathrm{K}$-index is a quasilogarithmic measure of the maximum fluctuations observed on a magnetometer measured at 3 hourly intervals. It's measured on an integer scale of 0-9. Normally its' values lies between 1 and 3, with 0 representing an unusually quiet period and $K$ values of 4-9 denoting magnetic storms.

\section{FSSC and FSGC indices}

Since K-indices are recorded every 3 hours (UTC), we can recognize differences in the initial phases of storms. There are two types of geomagnetic storms, those with sudden commencement and those with gradual commencement. Storms are therefore classified as ssc (Storms of Sudden Commencement) and sgc (Storms of Gradual Commencement). The details and dates of ssc and sgc are listed along with their K-indices at Kakioka Observatory. We limited our selection to events with $\mathrm{K}$-indices of 5 or more. We then counted the yearly frequencies of these events and named them FSSC (Frequency of Storms of Sudden Commencement) and FSGC (Frequency of Storms of Gradual Commencement) respectively.

\section{Data analysis}

We used wavelet analysis to study the effects of the new indices in details from a point of view of frequencies. Wavelet analysis illustrates the expansion of a time-series in terms of a set of basic functions. It expands functions in terms of wavelets (not trigonometric polynomials), that are generated in the form of translations and dilations of a fixed function called the mother wavelet.

Wavelet analysis is classified according to the selection of mother wavelets. In this paper, we used "Morlet" wavelet,
Figure 1 Variances of FSSC, FSGC and sunspot numbers

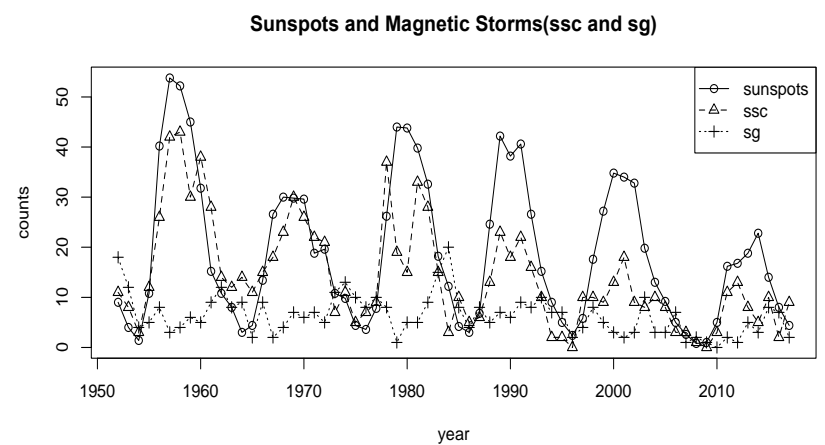

because it provided us a visualized $2 \mathrm{D}$ image of time and frequency. The efficiency of Morlet wavelet was shown for the study of solar activity[z2]. The results of Morlet wavelet analysis were expressed as wavelet coefficients $f(t, a)$, where $t$ represented time and ' $a$ ' a scale. The values $\mathrm{f}(\mathrm{t}, \mathrm{a})$ were transformed to color or gray scale in a 2D plane of time and scale (ta-plane). Our calculation of wavelets was performed using "R" with a wavelet package "sowas".

\section{RESULTS}

We defined new indices of geomagnetic fluctuation, FSSC(frequency of storm of sudden commencement) and FSGC(frequency of storm of gradual commencement) in the introduction. Fig.1 shows the variances of these indices compared with sunspot numbers.

We explored the correlations of these indices with 16 leading causes of death; tuberculosis, malignant neoplasms, diabetes mellitus, hypertensive diseases, heart diseases (excluding hypertensive heart diseases), cerebrovascular diseases, pneumonia, chronic bronchitis and emphysema, asthma, gastric ulcer and duodenal ulcer, diseases of liver, renal failure, senility, accidents, transport accidents, and suicide.

We first calculated the correlation coefficients between the yearly death rate from 16 leading causes of death and geomagnetic indices, K-index, FSSC, FSGC and the sunspot numbers. We also took into consideration a one year delay and indicated these as "FSSC $(\mathrm{d}=1)$ ". The results that were significant at the 95\% confidence level are listed in Table 1. The results of the sunspot numbers and FSGC are not listed because these were not statistically significant.

The FSSC index was positively correlated with hypertensive diseases, cerebrovascular diseases, asthma, gastric ulcer and duodenal ulcer, senility, accidents, and transport accident; and negatively correlated with malignant neoplasms, diabetes mellitus, heart diseases, pneumonia, chronic bronchitis and emphysema, renal failure. K-index was positively correlated with suicide, and negatively correlated with hypertensive diseases, cerebrovascular diseases, accidents, transport accidents.

In summary, FSSC has stronger correlation with most of fatality than sunspot numbers and K-index. There was no correlation with sunspot numbers. The fact that malignant neoplasms and heart diseases have negative correlation with FSSC suggests the effect of cosmic rays, because the effects 
Table 1 Correlations between causes of death and geomagnetic indexes

\begin{tabular}{|c|c|c|c|c|}
\hline Disease & k-index & $\begin{array}{l}\text { k-index } \\
(\mathrm{d}=1)\end{array}$ & FSSC & $\begin{array}{l}\text { FSSC } \\
(\mathrm{d}=1)\end{array}$ \\
\hline \multicolumn{5}{|l|}{ [Tuberculosis] } \\
\hline $\begin{array}{l}\text { [Malignant } \\
\text { neoplasms] }\end{array}$ & & & -0.389 & -0.387 \\
\hline [Diabetes mellitus] & & & -0.41 & -0.428 \\
\hline $\begin{array}{l}\text { [Hypertensive } \\
\text { diseases] } \\
\text { [Heart diseases ] }\end{array}$ & -0.253 & -0.249 & $\begin{array}{l}0.327 \\
-0.317\end{array}$ & $\begin{array}{l}0.347 \\
-0.283\end{array}$ \\
\hline $\begin{array}{l}\text { [Cerebrovascular } \\
\text { diseases] } \\
\text { [Pneumonia] }\end{array}$ & -0.331 & -0.336 & 0.362 & 0.348 \\
\hline $\begin{array}{l}\text { [Chronic bronchitis } \\
\text { and emphysema] } \\
\text { [Asthma] }\end{array}$ & & 0.234 & $\begin{array}{l}-0.309 \\
-0.457\end{array}$ & $\begin{array}{l}-0.302 \\
-0.46\end{array}$ \\
\hline & & & 0.307 & 0.278 \\
\hline $\begin{array}{l}\text { [Gastric ulcer and } \\
\text { duodenal ulcer] } \\
\text { ]Diseases of liver] }\end{array}$ & & & 0.287 & 0.281 \\
\hline [Renal failure] & & & -0.415 & -0.516 \\
\hline [Senility] & & & 0.351 & 0.31 \\
\hline [Accidents] & -0.303 & -0.236 & 0.275 & 0.324 \\
\hline [Transport accidents] & -0.369 & -0.327 & 0.323 & 0.396 \\
\hline [Suicide] & 0.331 & 0.293 & & \\
\hline
\end{tabular}

of cosmic rays are often accompanied by the decrease of geomagnetic activity. The link between the geomagnetic activity and the fatality of suicides and transport accidents have already been reported [8,9], and our results of FSSC affirm these reports. The positive correlation of asthma fatality with FSSC aligns with published literature [10].

Table 1 also shows the contradictory results among K-index and KSSC as for hypertensive diseases, cerebrovascular diseases, accidents, transport accidents. To explain this contradiction, we used the wavelet coherences. By selecting traffic fatality as a typical example, we first calculated the wavelet coherence between FSSC and traffic fatality. The result is shown in Fig.2.

Figure 2 Wavelet Coherence of FSSC-index and traffic fatality

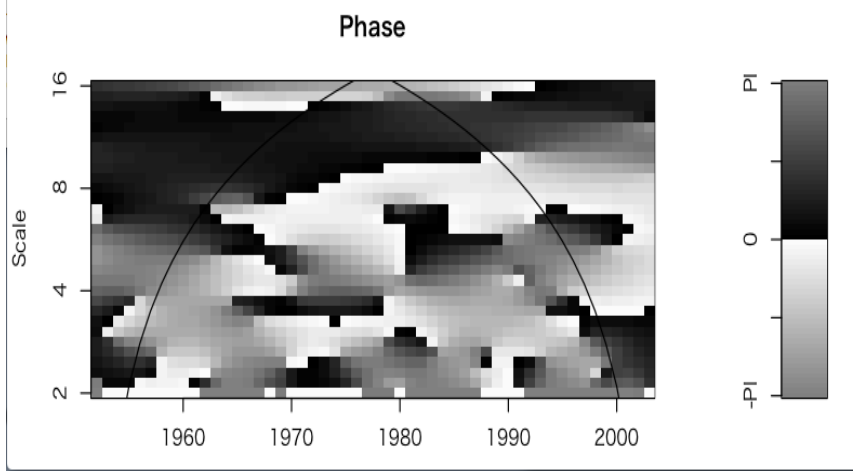

In Fig.2, the vertical values express the frequencies with unit "year". There is a blue long stripe around 9-years the phase of which is about pi/3. This implies the first variable FSSC leads the second variable Traffic fatality with the lag of 1.5 year.

Fig. 2 shows that the index FSSC precedes the traffic fatality around 9 years continuously from 1952 to 2004. This suggests the effects of FSSC on the traffic fatality with about one year lag.

We then calculated the wavelet coherence between K-index and the traffic fatality. The results are shown in Fig3.

Fig. 3 shows the wavelet coherence between K-index and the traffic fatality. The areas where $\mathrm{K}$-index preceded traffic fatality were found in various small areas, especially in frequencies less than 8 years.

The contradictory correlations found in Table 1 with respect to K-index and FSSC may result from the different features of wavelet coherences in Fig. 2 and 3. The details will be discussed again in the discussion section.

\section{DISCUSSION}

We defined new geomagnetic indices, FSSC and FSGC by separating the events of magnetic storms according to their types. We explored the variance of these two different types of magnetic storms, and found the link between the human health and FSSC, which could not been shown by usual indices such as sunspot numbers or K-index.

FSSC showed a statistically significant correlation with 13 causes of death, where 7 being positively correlated and 6 being negatively correlated with FSSC. On the contrary, The sunspot numbers showed no correlations with any 16 causes of death, and $\mathrm{K}$-index was correlated with only a few causes of death.

The negative correlation suggests the effects by cosmic ray to human health. When a geomagnetic storm is strong, it supplies a secure shield against cosmic rays. For example, the incidence of heart diseases increased after strong storms [5]

Our results of FSSC support this observation (Table 1). There were some contradictory results on correlations, when comparing the effects of K-index and FSSC. We examined the differences of the phase feature by

Figure 3 Wavelet coherence of K-index and traffic fatality
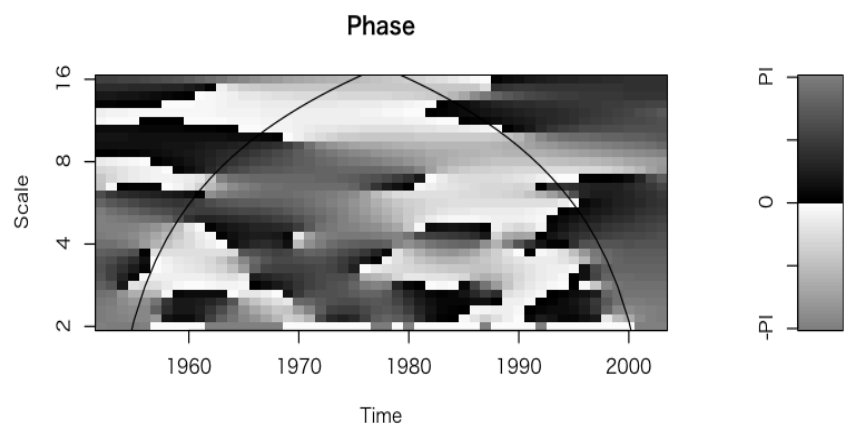
calculating wavelet coherences of K-index and FSSC (Fig.2 and 3). To explore further the difference of effects exerted by $\mathrm{K}$-index and FSSC more precisely, we calculated the wavelet coherence of both indices. In Fig.4, the first variable is FSSC, and the second is $\mathrm{K}$-index. Here, the positive value of phase implies that FSSC leads K-index.

Observing Fig.4, we can divide the correlation by its frequencies, and identify which factor leads the other. A positive value of phase implied that the FSSC (the first variable) preceded the $\mathrm{K}$-index (the second variable). In approximately 9 years of scales, FSSC leads K-index during the whole period from 1952 to 2004 . In less than 4 years of scales, there were areas where the K-index preceded FSSC. These results support our deduction about the cause of contradictory results.

For example, we encountered the contradictory results for the correlation of traffic fatality with FSSC and K-index. FSSC had positive phase compared with traffic fatality for about frequency of 11 years. Even for less than 8 years of scales, the phase is positive. On the contrary, K-index had a positive phase for only short durations around frequencies less than 8 years, and the lead of K-index was not strong enough. Subsequent to these observations, the K-index and FSSC have contradictory results as regards traffic fatality, because the phase is reversed for frequencies less than 8 years between K-index and FSSC. Generally speaking, traffic fatality was strongly related to FSSC, and FSSC preceded traffic fatality.

\section{Mechanism}

Melatonin synthesis plays an important role in circadian rhythms. Phase advance in pineal circadian rhythms is believed to be the underlying biochemical mechanisms that causes geomagnetic effects on human health [2]. Geomagnetic disturbances are linked to reduced nocturnal excretion of a melatonin metabolite in humans [11]. Abnormal melatonin levels are also seen in non-SAD (seasonal affective disorder) depression. This suggests that hydrophobic character of the cell membrane leads phase advance in some non-SAD depressive illness to changes in permeability [2].

Heart rate variability (HRV) reflects the state of the human nervous system's function. There is a link between geomagnetic activity and nervous system as shown from a continuous monitoring of HRV [12]. This supports our results regarding the relationship between FSSC and traffic fatality.

A major depression is activated with increased levels of IL-6 (interleukin-6) among other cytokines [13]. Since IL-6 plays an important role in human's immune system, this

Figure 4 Wavelet coherence of FSSC and K-index

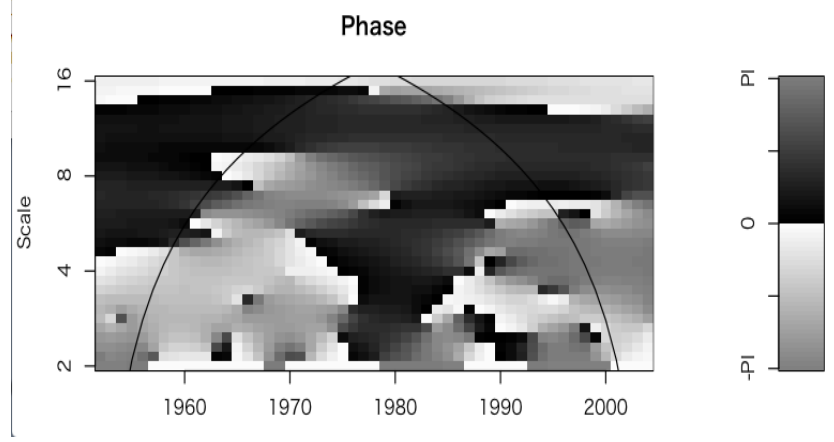

observation may reveal a new aspect of the effects of geomagnetic activity on human's immune system. These observations suggest the reason why the geomagnetic fluctuations have widespread effects on human health.

\section{CONCLUSION}

The new index FSSC (Frequency of Storms of Sudden Commencements) that was correlated to 13 of 16 leading causes of death in Japan between 1952 and 2004 supersedes sunspot numbers or K-index, and may provide a new clue that links geomagnetic activity and human health.

\section{REFERENCES}

[1] Palmer, S. J. Rycroft, M. and Cermack, J. M. (2006) Solar and geomagnetic activity, extremely low frequency magnetic and electric fields and human health at the Earth's surface. Surv Geophys, 27, 557-595, https://doi.org/10.1007/s10712-006-9010-7

[2] Kay, R.W. (1994) Geomagnetic Storms: Association with Incidence of Depression as Measured by Hospital Admission. British Journal of Psychiatry, 164, 403-409

[3] Morimoto.H. (2016) Exploring linkages between weather factors and the risk of cerebral infarction through the application of Bayesian networks, 2016, International Journal of Engineering and Applied Sciences (IJEAS), Vol.3, Issue 9, 14-17. ISSN: 2394-3661

[4] Cornélissen, G. (2002) Non-photic solar associations of heart rate variability and myocardial infarction. J. of Atmospheric and Solar-Terrestrial Physics, 64, Issues 5-6, 707-720, https://doi.org/10.1016/S1364-6826(02)00032-9

[5] Stoupel, E.(2017) Space Weather and Tachysystolic Sudden Cardiac Death. International Journal of Cardiology and Heart Health, 1, Issue 1, 09-11, https://doi.org/10.25141/2575-8160-2017-1.0009

[6] Gonzalez, W. D. Joselyn, J. A. Kamide,Y. Kroehl,H. W. Rostoker,G Tsurutani,B. T. and Vasyliunas,V. M. (1994), What is a Geomagnetic Storm?, J. Geophys. Res., 99(A4), 5771-5792.

[7] Fumio Maruyama, Kenji Kai, Hiroshi Morimoto (2017) Wavelet-based multifractal analysis on a time series of solar activity and PDO climate index. Advances in Space Research 60 1363-1372, http://dx.doi.org/10.1016/j.asr.2017.06.004

[8] Berk,M. Dodd,S. Henry,M. (2006) Do ambient electromagnetic fields affect behaviour? A demonstration of the relationship between geomagnetic storm activity and suicide. Bio Electro Magnetics, 27, Issue2, 151-155, https://doi.org/10.1002/bem.20190

[9] Dorman,L. I. (2005) Space weather and dangerous phenomena on the Earth: principles of great geomagnetic storms forcasting by online cosmic ray data. Annales Geophysicae, European Geosciences Union, 23 (9),2997-3002, HAL Id: hal-00317980, https://hal.archives-ouvertes.fr/hal-00317980

[10] Gadzhieva,G. D. and Rakhmatulinb,R. A. (2013) Influences of Solar and Geomagnetic Activity on Health Status of People with Various Nosological Forms of Diseases. Biophysics, 2013, Vol. 58, No. 4, pp. 568-572, https://doi.org/10.1134/S0006350913040040

[11] BBurcha,J. and SReifaM.GYostb,J.(1999) Geomagnetic disturbances are associated with reduced nocturnal excretion of a melatonin metabolite in humans. Neuroscience Letters, 266, Issue 3, 14, 209-212

[12] McCraty,R. Atkinson,M. Stolc, V. Alabdulgader,A.A. Vainoras,A. and Ragulskis,M. (2017) Synchronization of Human Autonomic Nervous System Rhythms with Geomagnetic Activity in Human Subjects. Int. J. Environ. Res. Public Health 2017, 14, 770, https://doi.org/10.3390/ijerph14070770

[13] Maes M, Ombelet W, De Jongh R, Kenis G, Bosmans E. (2001) The inflammatory response following delivery is amplified in women who previously suffered from major depression, suggesting that major depression is accompanied by a sensitization of the inflammatory response system. J Affect Disord 2001;63:85-92.

Hiroshi Morimoto Professor emeritus of Nagoya University. He graduated from Mathematical Institute of Nagoya University. He was first employed by Nagoya University as a pure Mathematician. Then he explored many fields including biology, global climate change, human health and bioweather, using methods of data mining. 